\title{
Motivations and Techniques for a Surface Detector to Veto Air Showers for Neutrino Astronomy with IceCube in the Southern Sky
}

\author{
The IceCube-Gen2 Collaboration ${ }^{\dagger}$, \\ ${ }^{\dagger}$ http://icecube.wisc.edu/collaboration/authors/icrc15_gen2 \\ E-mail: jauffenbeicecube.wisc.edu
}

\begin{abstract}
IceCube is currently the world's largest high-energy neutrino observatory, built at the geographic South Pole. For neutrino astronomy, a large sample of well-reconstructed astrophysical neutrinos of high purity is essential. The main backgrounds are muons and neutrinos, produced in cosmicray air showers in the Earth's atmosphere. The coincident detection of these air showers by the surface detector component IceTop is already used in IceCube neutrino analyses to veto atmospheric neutrinos and muons in the down-going region which at the South Pole corresponds to the southern sky. The successes and limitations of the IceTop array as an air shower veto motivate properties of future surface veto detectors to improve the performance of the next-generation neutrino telescope in Antarctica, IceCube-Gen2. The significance of this improvement depends on the features of the astrophysical neutrino spectrum. Here, we summarize different physics cases that motivate a surface veto and the motivation for different detection technologies based on first simulations and measurements.
\end{abstract}

Corresponding authors: J. Auffenberg*1

${ }^{1}$ RWTH Aachen University, Aachen, Germany.

The 34th International Cosmic Ray Conference,

30 July- 6 August, 2015

The Hague, The Netherlands

${ }^{*}$ Speaker. 


\section{Introduction}

The IceCube observatory [1] consists of 5160 photomultipliers deployed in depths between $1450 \mathrm{~m}$ and $2450 \mathrm{~m}$ into the Antarctic ice cap (deep detector, see Figure 1). IceCube reconstructs the trajectory of charged leptons produced during high-energy neutrino interactions in the surrounding ice via the emitted Cherenkov light. Recently published results show that IceCube has

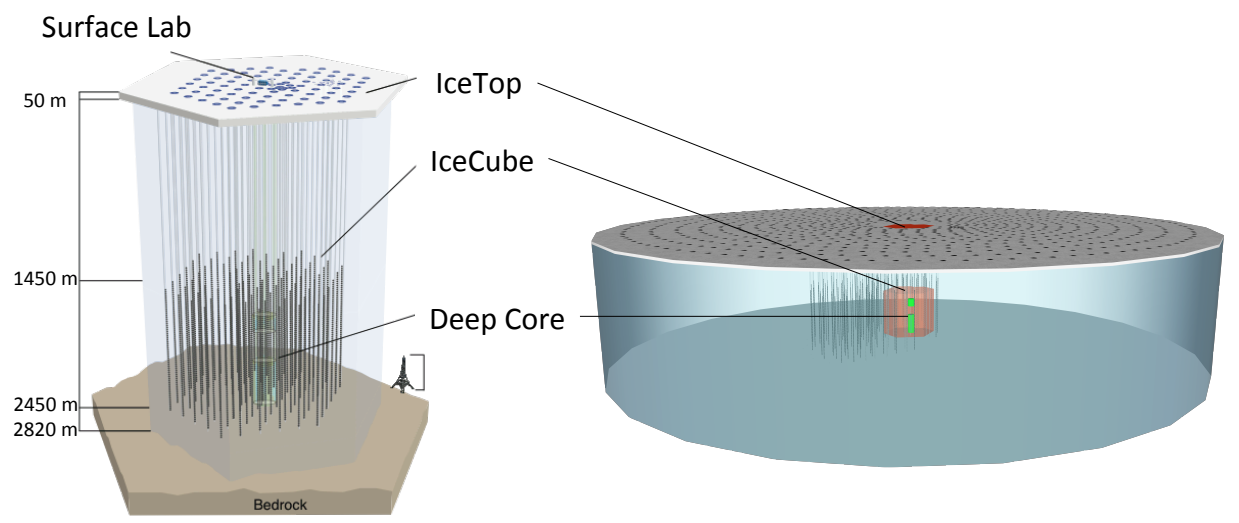

Figure 1: Artists view of IceCube and the IceCube-Gen2 detector with an extended surface array.

measured an astrophysical neutrino flux with high significance, however, no evidence for a point source has been found yet [2]. Above about $100 \mathrm{TeV}$ neutrino primary energy, the measured diffuse flux dominates the background of cosmic-ray-induced neutrinos and muons. One main task for the next-generation detector, IceCube-Gen2, will be the detection and high-quality reconstruction of a sufficient number of neutrinos for astronomical observations and a measurement of their spectrum with high precision (see also [3]).

The main backgrounds for extraterrestrial neutrinos are cosmic-ray induced particles produced in the atmosphere. One way to suppress some of these backgrounds is to select particles coming from the northern hemisphere, as charged particles are absorbed by the Earth. Thus only neutrinos (both astrophysical and atmospheric) reach the detector. In the southern hemisphere, the cosmicray-induced muon background dominates the neutrinos detected in IceCube because high-energy muons penetrate the several $\mathrm{km}$ overburden of ice above the detector. However, the corresponding cosmic-ray-induced air showers can be detected with a dedicated air shower array at the surface [4]. Such a surface detector can be used to veto the cosmic-ray-induced muons reaching the deep detector and is to some extent able to suppress even the atmospheric neutrino background. In fact, IceTop, an array of instrumented ice tanks located above IceCube, is already used in several analyses to suppress the atmospheric background, thereby increasing the sensitivity of IceCube for astrophysical neutrinos in the down-going region [5, 6, 7]. Another well-established way to distinguish the cosmic-ray-induced background from astrophysical neutrinos is to use the outer optical modules of the deep detector as veto [8]. Figure 2 shows the zenith distribution vs the energy deposit of the events in the deep detector found with this technique in four years of IceCube data using such a veto [2]. The corresponding event selection has two main features. 
First, it selects about 3.5 times more events with a dominating hadronic or electromagnetic cascade than events with a clear single muon track because most of the neutrino interactions do not produce elongated tracks. Second, IceCube does not detect any neutrinos with high light deposit in the up-going region (upper right corner of Figure 2), due to the neutrino cross section that grows with increasing energy. This shows that the detection of very high energy astrophysical neutrinos is limited to events that are down-going.

A veto at the surface of IceCube follows the idea to select starting events, but uses a surface detector to identify cosmic-ray-induced signals. As a result, one can also detect events that started in the volume between the deep detector and the surface array.

The advantages of detecting with a surface veto more high-energy astrophysical neutrinos containing a larger fraction of high-energy $v_{\mu}$-induced tracks are described in section 2 . Here, we discuss different motivations for surface extensions that lead to different possible configurations. In addition, different technological approaches for a cosmic-ray veto on the surface are summarized in section 3 .

\section{Motivation for surface detectors as veto for cosmic rays for IceCube-Gen2}

The idea for a surface veto is to rely on the construction of a hypothetical shower front based on the reconstruction of an event with the deep detector (see Figure 3). If one finds more than a certain number of hits in coincidence with the expected shower front in the surface detector, the event is vetoed as background for astrophysical neutrino detection.

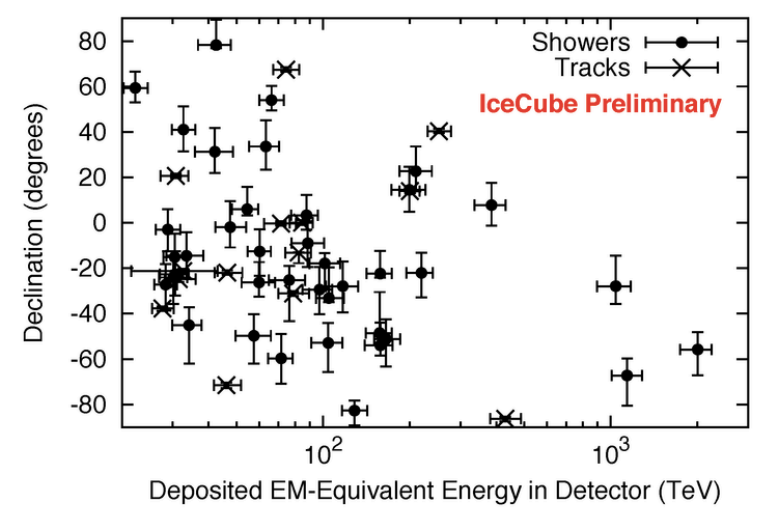

Figure 2: Zenith distribution vs. the energy deposit in the deep detector for the events found with the starting-event search after four years of data taking [2].

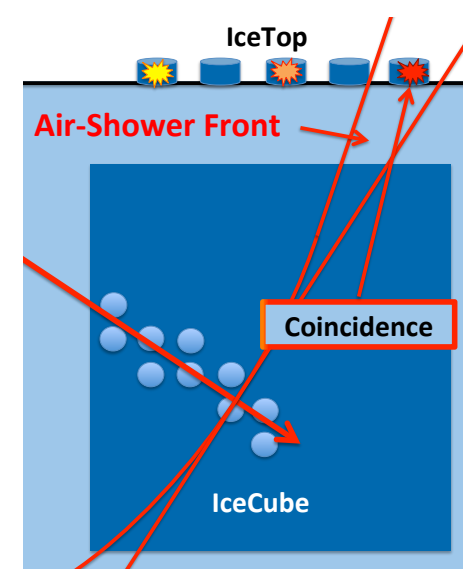

Figure 3: Schematic view of the IceTop veto strategy for the IceCube Observatory. If IceTop records hits consistent in time with a hypothetical shower front, the event is considered to be cosmic-ray-induced [9].

The general principle of such a surface veto is described in more detail in [9] with IceTop as an example. As mentioned in the introduction, IceCube-Gen2 extends the energy range of IceCube for the detection of astrophysical neutrinos and the identification of their sources [3]. In the following, the possible impact of a surface veto array on the measurement of different properties 
of the astrophysical flux are discussed and estimated. Detailed simulations of surface veto arrays will quantify the impact on these measurements in the future.

\subsection{Measurement of the properties of the diffuse spectrum}

Depending on the still-uncertain details of the astrophysical flux, the detection of astrophysical neutrinos in different energy regions can be of particular interest. For very soft spectra, it can be interesting to focus on moderate energies of as low as a few $\mathrm{TeV}$ to measure possible features of the neutrino spectrum. For harder spectra, the high-energy neutrinos are interesting, to measure a possible cut-off or change of slope at $\mathrm{PeV}$ energies. In both cases, a larger number of astrophysical neutrinos is needed.

At energies above $100 \mathrm{TeV}$, neutrinos from the northern sky are more and more likely to be absorbed during propagation through the Earth. It is therefore crucial to detect high-energy neutrinos from the southern sky (down-going). Here, an extended surface veto can improve significantly the detection of uncontained $v_{\mu}$-induced muon tracks. Table 1 shows the number of up-going and down-going astrophysical $v_{\mu}$ at energy thresholds of $1 \mathrm{TeV}, 10 \mathrm{TeV}$, and $100 \mathrm{TeV}$ neutrino energy. The numbers are based on Monte Carlo simulations. The injected flux was normalized to fit the observed astrophysical flux with 2 years of IceCube data [10]. The harder and steeper spectra account for the approximate uncertainty of the spectral index at the time. Up-going and horizontal $v_{\mu}$ up to $5^{\circ}$ above the horizon that interact in IceCube are not dominated by the background from muons that where produced in air showers. In the down-going region $\left(0^{\circ}-85^{\circ}\right.$ declination $)$ neutrino signatures in IceCube are several orders of magnitude fewer than penetrating muon tracks induced by cosmic rays. For example, for $100 \mathrm{TeV}$ vertical astrophysical neutrinos the muon rate is about 5 orders of magnitude higher than the expected astrophysical $v_{\mu}$ flux [7]. Here, an efficient surface veto detector would allow to obtain roughly the same number of $v_{\mu}$ above $10 \mathrm{TeV}$ neutrino primary energy from the down-going region as from the up-going region, and hence double the number of identifiable cosmic neutrinos almost independent from the spectral index. Due to the smaller observation region of $0^{\circ}-85^{\circ}$ declination in the southern sky, the background from atmospheric neutrinos ( $v_{\text {Atmos }}$ in table 1$)$ is $30 \%$ lower. Also the atmospheric neutrino background is expected to be vetoed to some extent.

The total number of astrophysical $v_{\mu}$ from the starting-event approach (Figure 2) and the number of astrophysical $v_{\mu}$ from the southern sky after applying a surface veto can be compared. The starting event search sacrifices about $40 \%$ of the in-ice detector for astrophysical neutrino detection due to the in-ice veto approach. After applying a surface veto this is not necessary anymore for the southern sky. In addition the active volume, where an astrophysical neutrino is allowed to interact, gets enlarged by the surrounding ice, now covered by the surface veto. Thus, for vertical events we gain $1.5 \mathrm{~km}$ ice above the detector in addition to the $40 \%$ increase from removing the in-ice veto. Overall the volume for astrophysical $v_{\mu}$ to interact and induce muon tracks increases by roughly a factor of 3.5 in the vertical direction. For the galactic center, a source candidate in the southern sky at $62^{\circ}$ declination, the overall gain in volume is roughly a factor of 8 [11]. The expected increase of high-energy tracks induced by astrophysical $v_{\mu}$ is similar and only slightly reduced due to absorption in the ice. These $v_{\mu}$ induced tracks are the event type with the most accurate pointing [12]. 


\begin{tabular}{||c|ccc||ccc||}
\hline & \multicolumn{3}{|c||}{ up-going } & \multicolumn{3}{c||}{ down-going } \\
\hline & $1 \mathrm{TeV}$ & $10 \mathrm{TeV}$ & $100 \mathrm{TeV}$ & $1 \mathrm{TeV}$ & $10 \mathrm{TeV}$ & $100 \mathrm{TeV}$ \\
\hline \hline$E^{-2}$ & 110 & 44 & 11 & 80 & 44 & 18 \\
\hline$E^{-2.3}$ & 220 & 60 & 9 & 160 & 57 & 13 \\
\hline$E^{-2.7}$ & 740 & 110 & 7 & 590 & 100 & 10 \\
\hline$v_{\text {Atmos }}$ & 15000 & 500 & 5 & 10500 & 350 & 5 \\
\hline
\end{tabular}

Table 1: Number of astrophysical neutrinos expected from the northern and the southern sky above different energy thresholds in IceCube in one year. The estimated event numbers are based on Monte Carlo simulations. The normalization derives from the measured astrophysical flux. Up-going is defined to end $5^{\circ}$ above the horizon, the region that is currently used in IceCube diffuse $v_{\mu}$ analyses. The down-going region is defined from the vertical to $85^{\circ}$ declination dominated by muons from air showers without a surface veto. $v_{\text {Atmos }}$ is the expected number of atmospheric neutrinos. No surface veto was applied to the $v_{\text {Atmos }}$.

\subsection{Identification of point sources}

Coincident hits in IceTop are a well established parameter in IceCube point source analyses to increase the sensitivity in the very down-going region $\left(\theta<25^{\circ}\right)$ [13]. In section 2.1 it was discussed that a surface veto could enable us to identify up to twice as many high-energy $v_{\mu}$-induced tracks compared to just the in-ice detector. They come with moderate energy resolution but sub-degree directional reconstruction [12]. The pointing of these highly significant neutrino events could be the key to identify sources of the astrophysical neutrino flux.

To first order the significance $\sigma=N_{\text {signal }} / \sqrt{N_{\text {signal }}+N_{\text {background }}}$ to identify a point source increases linearly with the number of signal events $N_{\text {signal }}$ in case of background domination. The denominator can further be approximated to decrease with $\sqrt{N_{\text {background }}}$. The number of background events $N_{\text {background }}$ is proportional to $\psi_{\text {event }}^{2}$, were $\psi_{\text {event }}$ is the angular uncertainty of the events. As a result, the significance of a point source search roughly increases linearly with the inverse of the angular uncertainty $\sigma \sim 1 / \psi_{\text {event }}$. Both the reduction of the cosmic-ray-induced background by orders of magnitude in the southern sky and the detection of more high-energy well reconstructed events will help to identify point sources in the southern sky.

The detection of a large number of almost background-free astrophysical neutrinos with subdegree pointing would enable us to produce a catalog of numerous sub-degree astrophysical neutrino directions. As due to absorption in the Earth most of the well-pointing high-energy events can not come from the northern hemisphere, such a catalog is particularly powerful for the southern sky. Assuming an isotropic distribution of sources in the sky, the total number of source candidates in the direction of the neutrino decreases again with the angular uncertainty $1 / \psi_{\text {event }}^{2}$. Thus the sensitivity of our experiment increases roughly with $1 / \psi$. This means that in first order one $v_{\mu}$ with a more than ten times better pointing compared to $v_{e}$ contributes more than ten times more to the significance to a point source search. This catalog of sub-degree neutrino directions can be used for various types of multimessenger analyses. One example would be dedicated follow up searches with gamma ray telescopes in these directions with the goal to find correlating source types.

\subsection{Detection of neutrinos with a cascade-like signature in IceCube}

As already discussed in the introduction of this section, most of the neutrino signals in IceCube 
are cascades. A large fraction of these cascades get produced by $v_{e}$ and $v_{\tau}$. Above about $1 \mathrm{PeV}$ the separation of $v_{e}$ induced cascades and $v_{t a u}$ induced signatures is possible [14]. In this case the $v_{e}$ and $v_{t a u}$ signatures are particularly interesting for analyses of the neutrino flavor composition. Furthermore, for $v_{\tau}$ with about $1 \mathrm{PeV}$ energy, the majority of detected events will be down-going as otherwise they would be absorbed in the Earth. From adding a surface veto, even if only efficient at $1 \mathrm{PeV}$, most of the background without any containment cut would get removed. As a result one can expect the number of $v_{\tau}$ to increase compared to the IceCube in-ice detector only. At 6.3 PeV the Glashow resonance, $\bar{v}_{e}+e^{-} \rightarrow W^{-}$rapidly increases the interaction cross-section for $\bar{v}_{e}$. At these energies $\bar{v}_{e}$ could be of particular interest for an extended surface array as for $v_{e}$ the containment arguments are similar to those from $v_{\tau}$.

\section{Hardware solutions for a surface veto}

A detector consisting of a large number of detection modules has to be very efficient in different ways:

- Low energy threshold and high detection efficiency increase the sensitivity to the neutrino flux.

- The number of astrophysical neutrinos detected due to the veto increases linearly with the duty cycle of a surface veto detector (exposure).

- One gains additional detection volume with increasing zenith as discussed in section 2.1.

- The system should be easily deployable and operable at low cost.

\subsection{Charged particle detection on the surface}

The most obvious option for a surface veto are particle detectors that measure the Cherenkov or scintillation light produced by charged particles in an enclosed active volume like tanks or paddles. Results from first Monte Carlo simulations that are based on shower parameterizations underline the importance of the total collection area and sensitivity to both the electromagnetic and the muonic part of the shower [15]. First estimates of the veto efficiency of a particle detector on the surface, based on a geometry described in [11], show more than $99 \%$ veto efficiencies at $\sim 20 \mathrm{PeV}$ cosmic ray primary energy at $\cos (\theta)=0.5$ (see Figure 4). To get higher veto efficiencies or lower passing rates for cosmic rays, a denser array could be considered (Figure 5). A detailed investigation of the event signatures and rates induced by cosmic-rays in the in-ice detector and subsequent comparison to those predicted from the astrophysical neutrino flux will show the potential of this geometry for astrophysical neutrino searches. Uncertainties due to particle density fluctuations in the air shower will need careful investigation.

\subsection{Shower detection in the atmosphere}

An alternative approach to cosmic ray shower particle detectors are detection systems that do not use a separated active volume as a calorimeter but the entire atmosphere above the detector. The radio emission of air showers in the atmosphere and the air Cherenkov emission or fluorescence light emission are examples of such signals. 


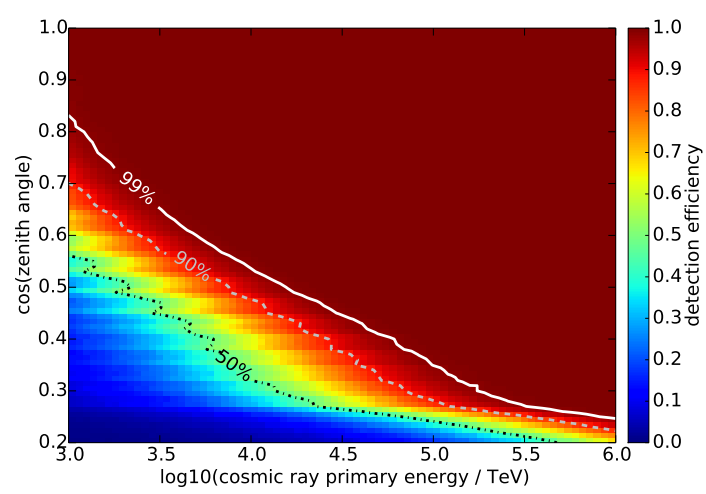

Figure 4: Veto efficiency as a function of zenith angle and cosmic ray primary energy for a surface detector as shown in Figure 1 right. The estimate is based on combined parameterizations of the lateral particle densities in air showers for muons and electrons.

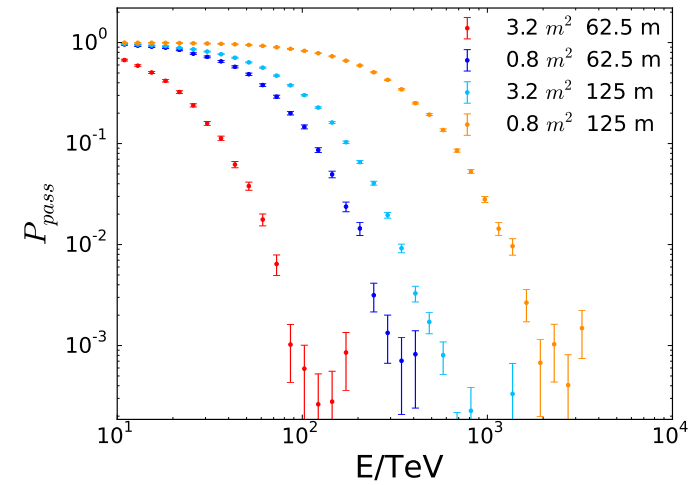

Figure 5: Passing fraction of quasi-vertical air showers $\left(<25^{\circ}\right)$ from CORSIKA simulations. Array configurations that have a spacing of $62.5 \mathrm{~m}$ or $125 \mathrm{~m}$ and a scintillator surface area of $0.8 \mathrm{~m}^{2}$ or $3.2 \mathrm{~m}^{2}$ are shown.

The radio emission of air showers is a small signal in the regime of some $\mathrm{MHz}$ to several hundred $\mathrm{MHz}$ and can only be detected above the electromagnetic background at cosmic ray primary energies above $10 \mathrm{PeV}$. Therefore, this detection technique is currently not under further investigation for a veto detector [16].

The emission of Cherenkov light of cosmic-ray induced air showers is a rather strong signal compared to the night sky background. It can be detected down to much lower primary energies compared to other detection techniques. Assuming that an air Cherenkov detector has to work efficiently as a veto at about $100 \mathrm{TeV}$ primary energy, only relatively small telescopes with apertures $<1 \mathrm{~m}$ are required. The detection efficiency for cosmic rays, and related, the density of detectors that are necessary for a veto detector, need to be investigated for air Cherenkov telescopes. Another challenge for air Cherenkov detectors at the South Pole are the environmental conditions. While extreme cold conditions can be simulated in laboratories, the influence of many other conditions of the Antarctic weather on the performance of such telescopes have to be investigated at the South Pole. For this purpose, a prototype telescope was developed that will be tested at the South Pole in the 2015/16 summer season [17]. The analyses of LIDAR observations at the South Pole show promising results concerning the estimated up-time of a telescope for air shower detection [18]. The data suggests an overall up-time as high as $20-25 \%$ of the year might be possible (with $50 \% \pm 10 \%$ up-time during the austral winter).

\section{Summary}

We have shown that a surface detector optimized for vetoing muons that penetrate the in-ice detector of IceCube-Gen 2 could significantly enhance the number of detectable astrophysical neutrinos. At energies above $10 \mathrm{TeV}$, a surface veto could enable IceCube-Gen2 to detect as many down-going astrophysical $v_{\mu}$ as are currently detected as up-going. This ratio increases with the 
energy threshold. Improvements in the measurement of different properties of the astrophysical neutrino flux were motivated. The detection of neutrinos with very good pointing on a very low background would increase the sensitivity of point source searches. Detectors based on particle detection in enclosed volumes are an obvious technology for surface air shower detectors. Alternative detection methods like air Cherenkov telescopes are under investigation. Detailed simulations will determine the impact of a surface detector to veto cosmic ray signals on the scientific goals of IceCube-Gen2.

\section{References}

[1] A. Achterberg et al. (IceCube Collaboration), Astropart. Phys., 26 (2006) 155-173, doi: 10.1016/j.astropartphys.2006.06.007.

[2] IceCube Coll., Observation of Astrophysical Neutrinos in Four Years of IceCube Data, PoS(ICRC2015)1081 these proceedings.

[3] IceCube Coll., IceCube-Gen2 High Energy Array, PoS(ICRC2015)1146 these proceedings.

[4] R. Abbasi et al. (IceCube Collaboration), Nucl. Inst. Meth. A, 700 (2013) 188-220, doi: 10.1016/j.nima.2012.10.067.

[5] IceCube Coll., A search for extremely high energy neutrinos in 6 years of IceCube data, PoS(ICRC2015)1064 these proceedings.

[6] IceCube Coll., Results of neutrino point source searches with 2008-2014 IceCube data above $10 \mathrm{TeV}$, PoS(ICRC2015)1047 these proceedings.

[7] IceCube Coll., IceTop as Veto for IceCube, PoS(ICRC2015)1086 these proceedings.

[8] S. Schönert, T. K. Gaisser, E. Resconi, and O. Schulz, Phys. Rev. D 79, 043009 (2009), doi: 10.1103/PhysRevD.79.043009.

[9] IceCube Coll., IceTop as a veto in astrophysical neutrino searches for IceCube, paper 0373 ICRC 2013 proceedings, http://arxiv.org/pdf/1309.7010.pdf.

[10] M. G. Aartsen et. al. (IceCube Collaboration), Science 342 (2013), doi: 10.1126/science.1242856.

[11] IceCube Coll., IceVeto: An extension of IceTop to veto horizontal air showers, paper 0374 ICRC 2013 proceedings, http://arxiv.org/pdf/1309.7010.pdf.

[12] M. G. Aartsen et. al., JINST 9 (2014), P03009, doi: 10.1088/1748-0221/9/03/P03009.

[13] M. G. Aartsen et. al. (IceCube Collaboration), ApJ 779132 (2013), doi: 10.1088/0004-637X/779/2/132.

[14] IceCube Coll., A Search for Astrophysical Tau Neutrinos in Three Years of IceCube Data, PoS(ICRC2015)1071 these proceedings.

[15] IceCube Coll., Simulation Studies for a Surface Veto Array to Identify Astrophysical Neutrinos at the South Pole, PoS(ICRC2015)1070 these proceedings.

[16] J. Auffenberg et. al., A radio air shower surface detector as an extension for IceCube and IceTop, paper 0293 ICRC 2007 proceedings, http://arxiv.org/abs/0708.3331.

[17] J. Auffenberg et. al., Design Study of an Air Cherenkov Telescope for Efficient Air-Shower Detection at 100 TeV at the South Pole on Top of IceCube, PoS(ICRC2015)1155 these proceedings.

[18] S. Benzvi et. al., An Estimate of the Live Time of Optical Measurements of Air Showers at the South Pole, PoS(ICRC2015)568 these proceedings. 